\title{
Ärztliche Arbeit in der dritten Welt - eine Alternative
}

\author{
Auch wenn in der Schweiz schon die Rede ist von einem zukünftigen Ärzte- \\ mangel, sei daran erinnert, dass in der dritten Welt vielerorts die medizini- \\ sche Versorgung der Bevölkerung miserabel ist. Gelegenheiten, dort sich zeit- \\ weilig zu engagieren und Aufbauarbeit zu leisten, gibt es immer noch: eine \\ faszinierende Aufgabe und ein Appell an unsere Solidarität!
}

\section{Hans Güntert*}

* Der Autor ist seit kurzem Mitglied des Verwaltungsrates der Fondation médicale Ad lucem au Cameroun. Im Text wurde für Personen beider Geschlechter gelegentlich nur die männliche Form verwendet - dies ausschliesslich aus Gründen der Lesbarkeit.
Korrespondenz:

Dr. med. Hans Güntert Junkerstrasse 2 CH-9500 Wil
Eigene Erfahrungen seit mehr als 40 Jahren veranlassen mich, in der Schweizerischen Ärztezeitung auf das Thema bzw. auf die Möglichkeiten ärztlicher Tätigkeit in der dritten Welt aufmerksam zu machen und sie als wertvolle Alternative jedenfalls für eine gewisse Zeit während des Berufslebens darzustellen. Der Sprung über unsere Grenzen hinaus in die armen Länder des Südens zeigt, dass vieles ganz anders ist als bei uns. Kein Wunder auch, dass es einen demographischen Druck gibt von der ärmeren Welt hin zur reicheren des Westens mit allen daraus resultierenden Problemen. Wir wissen, dass wir in vieler Hinsicht privilegiert sind.

\section{Medizin hier - Medizin dort}

Der Abstand zwischen unserer Medizin und derjenigen in der dritten Welt ist - abgesehen von Ausnahmen (private Institutionen für die Wohlhabenden) - ein gewaltiger, entsprechend den allgemein vorhandenen Standardunterschieden sozusagen in allen Lebensbereichen (Abb. 1). Während bei uns die obligatorische Krankenversicherung eine sehr gute Grundversorgung gewährleistet, ist in den armen Ländern für viele Menschen der Zugang zu den medizinischen Einrichtungen schwierig, wenn nicht gar unmöglich. Es fehlen allzu häufig die finanziellen Mittel. Systeme der sozialen Sicherheit sind in diesen «schwachen» Staaten meist inexistent. Die Frage stellt sich, ob wir uns von dieser Situation berühren lassen. Ich wage zu hoffen, dass viele unserer Berufsangehörigen die Überzeugung in sich tragen, dass unser Planet einen Abbau der Nord-Süd-Gegensätze braucht. Ist es nicht sinnvoll und vielleicht sogar dringlich, auch in diesem Sinn an unseren materiellen Überfluss Fragen zu stellen? Natürlich können alle Ärzte der Welt die grossen Gegensätze nicht beseitigen. Aber jeder persönliche ärztliche Einsatz leistet dazu einen Beitrag.

\section{Travail médical au tiers monde - une alternative}

Nombreux sont les pays du tiers monde, où les services de santé sont encore sous-développés. Ça correspond a un niveau de vie bas tout en général pour la plupart des gens. L'auteur, qui a une expérience au Cameroun de cinq ans (1962-1967!), $s^{\prime}$ occupe depuis quelque temps de nouveau de ces problèmes. II explique, qu'une reprise d'une coopération plus étroite est utile surtout pour les pays les plus pauvres, mais aussi enrichissante sous plusieurs points de vue pour les médecins qui sont prêts à s'engager temporairement - plutôt avec une formation déjà avancée. Le texte veut être un encouragement pour cette alternative. II montre aussi I'importance de l'attitude des médecin-chefs, qui peuvent rendre difficile un départ ou le faciliter.

Solche Einsätze aus der Schweiz waren in den 60er und bis in die 80er Jahre des letzten Jahrhunderts recht zahlreich - ich bin der Ansicht, dass es gut wäre, sie würden sich jetzt auch wieder mehren. Dafür gibt es gute Argumente: Eines ist gewiss die vielfache Not in bezug auf die Gesundheitsversorgung in den Ländern der dritten Welt. Ein anderes sehe ich in den positiven beruflichen und menschlichen Erfahrungen, die in einer wesentlich anderen Umwelt gemacht werden: Horizonterweiterung, Kennenlernen anderer Mentalitäten, Kulturen und Verhaltensweisen, Arbeit mit einfachen Mitteln. Man steht auch einer andern Pathologie gegenüber. Die eigentlichen Tropenkrankheiten sind vielerorts verbreitet und bedrohen zahlreiche Menschenleben. Dasselbe gilt für die Folgen mangelnder 


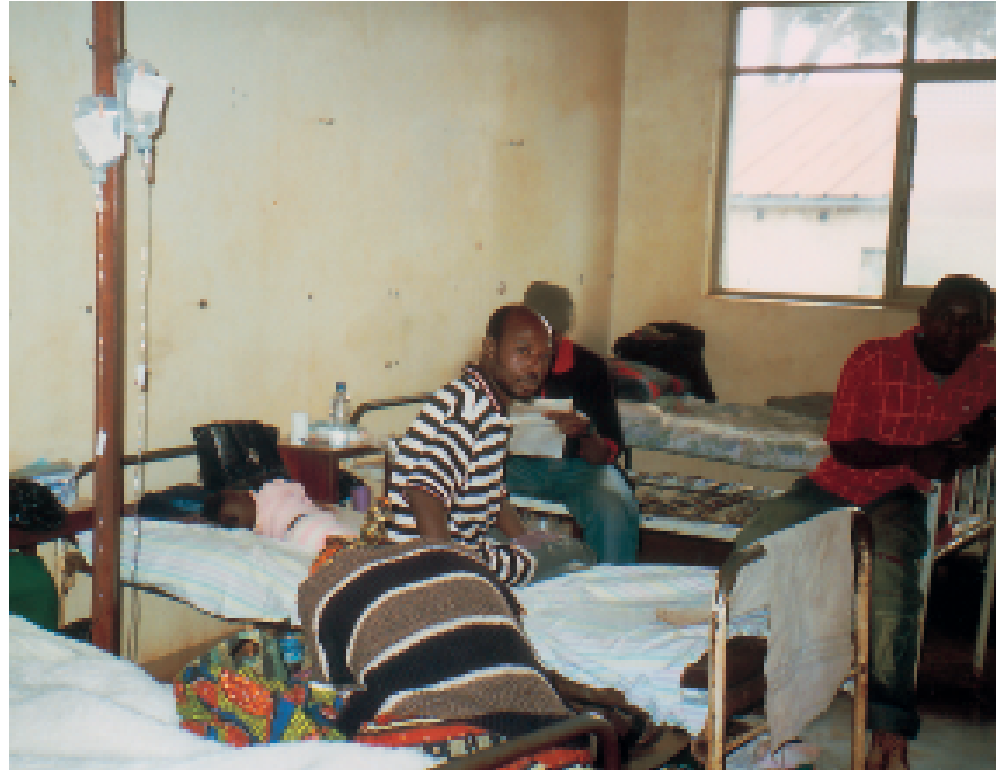

Jeder Patient hat mindestens einen Angehörigen bei sich.

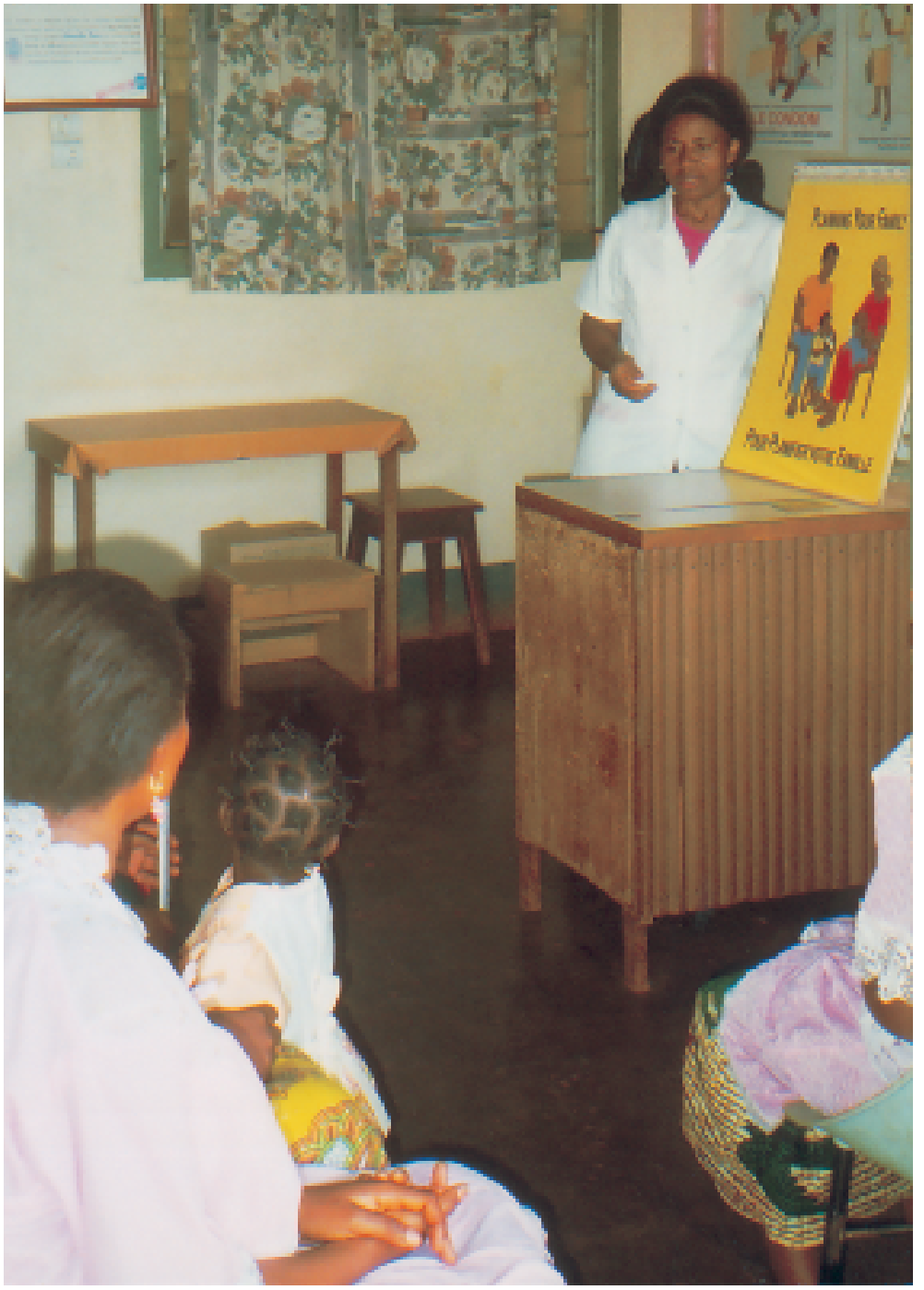

Familienplanung ist am besten durch Einheimische zu vermitteln.
Hygiene. Bei uns seltene Infektionskrankheiten können epidemisch auftreten und den Fortbestand ganzer Bevölkerungsteile bedrohen (Aids). Typisch ist, dass bei vielen Krankheiten die Patienten erst in einem fortgeschrittenen Stadium zum Arzt gehen, was die Diagnostik oft sehr vereinfacht. Die Anamnese - häufig mit Hilfe eines Dolmetschers aufzunehmen - und der klinische Befund haben eine unvergleichlich grössere Bedeutung als bei uns, wo möglichst alles medizintechnisch erhärtet sein muss. Die vielen vital bedrohlichen Zustände verlangen rasches therapeutisches Handeln - eine Schule der Entschlussfähigkeit. Ganz allgemein haben die Medizinalpersonen nicht nur kurativ zu handeln, sondern auch der Prävention ihre volle Aufmerksamkeit zu schenken, dies weniger durch eigene Aktivität als bei der Instruktion und Organisation entsprechender Programme. Dies setzt aber bereits eine gute Vertrautheit mit den örtlichen Gegebenheiten voraus. Besondere Behutsamkeit ist geboten, wo es um die Veränderung von Lebensgewohnheiten (Beispiel Familienplanung: Abb. 2) geht. Zur Bereitschaft, in vielen Bereichen tätig zu werden, gehört auch die Ausbildungsfunktion, sei es für das Pflegepersonal, für Studenten oder frischgebackene Ärzte. Viele von diesen verschiedensten Erfahrungen können auch nach der Rückkehr aus einem Entwicklungsland hier sehr gut verwertet werden.

Es entzieht sich meiner Kenntnis, ob heute noch eine grössere Anzahl von Jungmedizinern an eine zeitweilige Arbeit in einem Entwicklungsland denkt. Eine grundsätzlich positive Einstellung zur Entwicklungszusammenarbeit und auch eine gewisse Risikofreude sind gute Voraussetzungen. Nicht zufällig sind etliche Ärzte in der Schweiz heute in leitenden Stellungen tätig, die in ihren jüngeren Jahren Wert und Sinn der Arbeit in einer benachteiligten Weltgegend erkannt und erfahren haben.

Wichtig sind die Fragen nach Zeitpunkt und Dauer eines Einsatzes innerhalb der Weiterbildungsphase. Wer sich für die Arbeit in einem Entwicklungsland interessiert und sich gründlich informiert, wird gut daran tun, zwei Jahre oder auch mehr einzuplanen, und zwar nicht am Anfang, sondern am Ende der Weiterbildung. Wo immer eine Fachkraft in einer primär unvertrauten Welt sich einfügen und engagieren will, benötigt sie zum vornherein rund ein halbes Jahr, bis sie sich auskennt und bis ein gegenseitiges Vertrauen aufgebaut ist. «Voir, comprendre, agir» gilt immer noch als kluge Verhaltensweise, die das Mögliche mit Hilfe gutinstruierter lokaler Kräfte zu erreichen sucht, z.B., um in irgendeinem mangelhaften Bereich Verbesserungen zu 


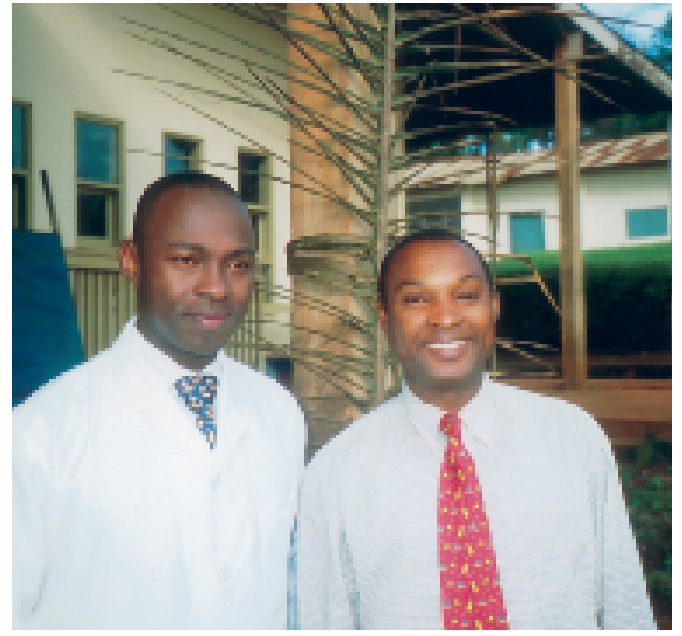

Zwei Kameruner - ein Allgemeinmediziner (li) und ein Gynäkologe (re).

erzielen. Diese Verbesserungen müssen freilich auch von den Einheimischen verstanden und bejaht werden. Dass auch kürzere Einsätze öfters durch junge Kräfte meist in akuten Notsituationen schweren Grades (Krieg, Naturkatastrophen) nützlich sind, ist ebenso richtig und wichtig, wie die zwar selteneren Engagements von älteren, erfahrenen Kollegen, die nochmals eine besondere Herausforderung suchen.

\section{Und das einheimische Fachpersonal?}

Grosse Fragen stellen sich in bezug auf das Vorhandensein oder den Mangel an einheimischem ärztlichem und seltener auch von Pflegepersonal. Hier bestehen von Land zu Land erhebliche Unterschiede. Medizinische Fakultäten sind vielerorts in der zweiten Hälfte des letzten Jahrhunderts errichtet worden. Das bedeutet noch nicht, dass damit die ärztliche Versorgung gesichert sei. Wegen der schon erwähnten fehlenden Strukturen wie Krankenversicherungen und mindestens ebenso wegen der verbreiteten Armut sind die finanziellen Voraussetzungen für die landeseigenen Ärzte oft nicht verlockend, ebenso nicht die Ausbildungsmöglichkeiten (Abb. 3) Die möglichen Konsequenzen für die Einheimischen liegen auf der Hand: Die Abwanderung in westliche Länder ist verbreitet, wo die Erlangung eines Spezialistendiploms und ein besseres Einkommen winken. Allzu häufig wird die Auswanderung in ein reiches Land zu einem Definitivum (brain drain) - der eigenen Bevölkerung ist damit allerdings wenig gedient, vielleicht am ehesten noch der Familie ... Nichtstaatliche medizinische Einrichtungen, die auch heute noch häufig irgendwie den verschiedenen Kirchen oder andern religiösen Gruppierungen nahestehen bzw. teilweise von diesen getragen werden und die grundsätzlich auch den armen Anteilen der Bevölkerung dienen wollen, haben deshalb heute wieder vermehrten Ärztemangel. Um so wichtiger kann deshalb unsere Präsenz werden.

\section{Ein Beispiel}

Ein mir vertrautes Arbeitsfeld sei kurz beschrieben: Das Ad-lucem-Spital von Mbouda in Kamerun wurde 1957 gegründet und machte lange eine erfreuliche Entwicklung durch. Es gehört einer grösseren Organisation an, die sich «Fondation médicale Ad lucem au Cameroun» nennt. $\mathrm{Zu}$ ihr gehören 10 Spitäler und 22 Dispensaires mit einem Personalbestand von über 800 Mitarbeitenden, wovon 20 Ärzte (zz. keine Europäer!). Während in den ersten drei Jahrzehnten die Entwicklung in baulicher und fachlicher Hinsicht stetig voranschritt bei während langer Zeit möglicher Selbstfinanzierung (!), begann sich die wirtschaftliche Situation des Landes $1985 \mathrm{zu}$ verschlechtern. Die schliesslich totale Kamerunisierung der obersten Leitung führte zu einer zunehmend mühsamen Periode, die den Rückzug aller Europäer zur Folge hatte. Eine gewisse Wende setzte ab 2002 ein, als die Verantwortlichen erkannten, dass sie aus eigener Kraft den Betrieb kaum mehr aufrechterhalten konnten. Ein Appell an die Gruppe ehemals dort tätiger europäischer Ärzte und der Zugang zu einer hiesigen Finanzquelle erlaubt dem Spital, jetzt wieder besser zu arbeiten. Der Chefarzt jedoch, der die chirurgische Abteilung betreut, hat bei total bis 200 hospitalisierten Patienten und relativ viel Personal in Ausbildung (u.a. auch Studenten) nur noch einen älteren Kollegen zur Seite, der sich praktisch auf Ultraschalluntersuchungen beschränkt. Nicht wenig eigentliche ärztliche Arbeit wird von «Assistants» geleistet, die im Lauf der Jahre als erfahrene Pflegekräfte «nachgenommen» wurden und z.T. sogar operativ tätig sind. In diesem Spital fänden ein Internist und ein Pädiater eine dankbare Aufgabe. Weitere europäische Spezialisten (Abb. 4) sind auch für andere Ad-lucem-Spitäler gesucht und erwünscht.

\section{Anregung und Ermutigung}

Liebe Kolleginnen und Kollegen, denken Sie auch an diese Alternative der Arbeit in der dritten Welt, wenn es darum geht, Ihren Lebensplan zu entwerfen. Natürlich ist eine frühe Kontaktnahme mit einer der Organisationen (siehe unten), die Ärzte zum Einsatz bringen, unerlässlich, ebenso die Beschäftigung mit entwicklungspolitischen Gegebenheiten. Dass das Engagement bei bestehender Partnerschaft gemeinsam getragen und bejaht werden muss, ist eine 
Selbstverständlichkeit. Sehr hilfreich und wichtig für Interessierte wird das Verständnis und die Mitwirkung des zuständigen Chefarztes in der Schweiz sein. In diesem Zusammenhang stellt sich vielleicht sogar die Frage nach der Wiedereintrittsmöglichkeit in die Heimatklinik. Ich kann hier nur an die Chefärzte appellieren, ihre

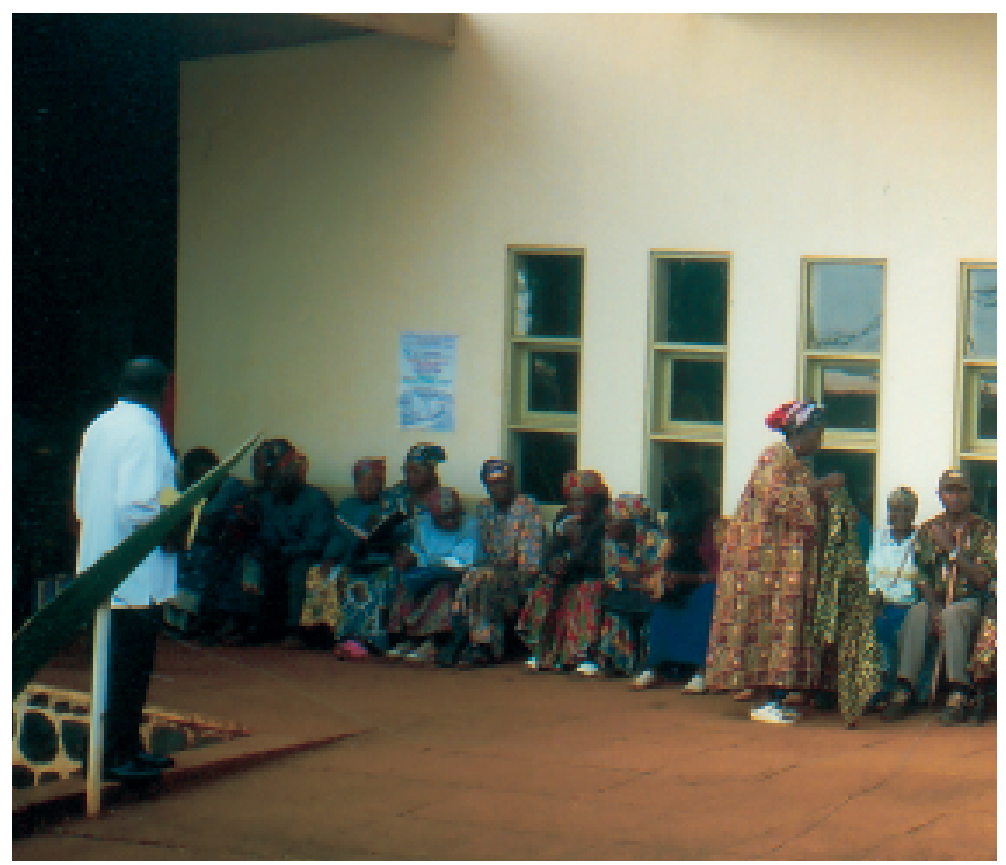

Der europäische Augenspezialist aus einem andern Zentrum hält seine monatliche Sprechstunde. besonders mutigen Mitarbeiter zu unterstützen, freizugeben und bei Bedarf auch wieder einzugliedern. Eine Ausreise und der Einsatz ist dann von der nicht unbedeutenden Sorge um das «Nachher» befreit.

Wenn eine grössere Anzahl von Schweizer Ärzten sich in armen Ländern engagieren würde, wäre dies ein guter Beitrag für die «eine Welt». Ärzte dort können ein wertvolles Vorbild sein, wenn sie sich menschlich und beruflich bewähren. Vielleicht bewegen sie den einen oder andern einheimischen Kollegen, seinem Land treu zu bleiben und nicht in den angenehmeren Westen zu fliehen. In die Schweiz Zurückgekehrte werden zu einem besseren völkerverbindenden Verständnis beitragen.

Herrn Dr. W. Munz, Wil, danke ich für die Durchsicht und seine Anregungen.

Weitere Informationen erhalten Sie u.a. bei den folgenden Organisationen:

- Medicus Mundi Schweiz, Murbachstrasse 34, Postfach, 4013 Basel, info@medicusmundi.ch;

- Médecins sans frontières, Postfach, 8030 Zürich, www.msf.ch;

- Mission 21, Evangelisches Missionswerk, Missionsstrasse 21, 4003 Basel, info@mission21.org;

- Schweizer Hilfsverein für das Albert-Schweitzer-Spital Lambarene, Champ Carré 9, 1256 Troinex, info@schweitzer.org;

- Solidarmed, Obergrundstrasse 17, Postfach, 6000 Luzern 4, solidarmed@solidarmed.ch. 